PROCEEDINGS OF THE

AMERICAN MATHEMATICAL SOCIETY

Volume 129, Number 4, Pages 989-998

S 0002-9939(00)05686-0

Article electronically published on October 4, 2000

\title{
A FUNCTIONAL EQUATION ARISING FROM RANKED ADDITIVE AND SEPARABLE UTILITY
}

\author{
JÁNOS ACZÉL, GYULA MAKSA, CHE TAT NG, AND ZSOLT PÁLES \\ (Communicated by Jonathan M. Borwein)
}

\begin{abstract}
All strictly monotonic solutions of a general functional equation are determined. In a particular case, which plays an essential role in the axiomatization of rank-dependent expected utility, all nonnegative solutions are obtained without any regularity conditions. An unexpected possibility of reduction to convexity makes the present proof possible.
\end{abstract}

\section{INTRODUCTION}

The problem of axiomatizing preferences between uncertain binary alternatives in which an additive representation holds over consequences when the chance event is held fixed and a separable (product) representation holds between consequences and events was raised by R. D. Luce [8]. Assuming that both consequences have the same separable representation, the problem reduced to solving the functional equation

$$
f(v)=f(v w)+f(v q(w)) \quad(v \in[0, k[, w \in[0,1])
$$

with the unknown functions $f:[0, k[\rightarrow[0,+\infty[(k \in] 0,+\infty])$ and $q:[0,1] \rightarrow[0,1]$. While it is quite natural to assume that $f$ is strictly increasing, mapping the domain $[0, k$ [ onto an interval $[0, K[$, and $q$ is strictly decreasing and onto $[0,1]$, equation (11) has been completely solved by J. Aczél, R. Ger and A. Járai [3] without any such assumption. They found that the following is the complete list of solutions:

$$
\begin{gathered}
f(v) \equiv 0, \quad q:[0,1] \rightarrow[0,1] \text { arbitrary; } \\
f(v)=\alpha \quad(v \in] 0, k[), \quad f(0)=0, \\
q(w)=0 \quad(w \in] 0,1]), \quad q(0) \in] 0,1] \text { arbitrary; }
\end{gathered}
$$

Received by the editors June 7,1999 .

2000 Mathematics Subject Classification. Primary 39B12, 39B22, 39B72; Secondary 26A48, 26A51, 91A30, 91C05.

Key words and phrases. Functional equation, binary gamble, rank-dependent expected utility, convexity.

This research was supported in part by the Natural Sciences and Engineering Research Council (NSERC) of Canada Grants OGP 0002972 and OGP 0008212, by the Hungarian National Science Foundation (OTKA) Grant T-030082 and by the Higher Education Research Council (FKFP) Grant 0310/1997.

The authors are grateful to R. Duncan Luce for communicating the problem and explanations and to the referee for pointing out a confusing misprint in the first version of the manuscript. 
and

$$
\begin{gathered}
f(v)=\alpha v^{c} \quad(v \in[0, k[), \\
q(w)=\left(1-w^{c}\right)^{1 / c} \quad(w \in[0,1]) \quad(\alpha>0, c>0 \text { constants }) .
\end{gathered}
$$

Luce first thought that this provided an axiomatization of rank-dependent expected utility $(R D E U)$, whereas in reality it axiomatized only the stronger case of rank-independent expected utility. Exploring more general representations, R. D. Luce and A. A. J. Marley [9] noted that if one assumes different separable (product) representations for the two consequences whose utilities are related by a strictly increasing and surjective function $g$, one can proceed as follows.

One assumes that the expected utility $U(x, C ; y, \bar{C})$ from a gamble $(x, C ; y, \bar{C})$ (the event $C$ has consequence $x$, the complementary event $\bar{C}$ has consequence $y$ ) allows the representation

$$
f_{0}[U(x, C ; y, \bar{C})]=f[u(x) W(C)]+f_{1}\left[u_{1}(y) W_{1}(\bar{C})\right],
$$

where $f_{0}, f, f_{1}:\left[0, k\left[\rightarrow\left[0,+\infty\left[, f_{0}(0)=f_{1}(0)=0\right.\right.\right.\right.$, the functions $W, W_{1}$ map the set of events onto $[0,1]$ and $u$ maps the set of consequences onto $[0, k[$. Furthermore, as mentioned above, $u_{1}=g \circ u$, where $g:[0, k[\rightarrow[0, k[$ is a surjective ("onto") strictly monotonic function. Generalizing $W_{1}(\bar{C})=1-W(C)$, also $W_{1}(\bar{C})=$ $q(W(C))$ is assumed $(q:[0,1] \rightarrow[0,1]$; no monotonicity or surjectivity postulated). If $\emptyset$ denotes the empty and $E$ the universal event, then $W(\emptyset)=W_{1}(\emptyset)=0$ and $W(E)=W_{1}(E)=1$. Since $(x, C ; x, \bar{C}) \sim x(x$ is the consequence whatever event occurs), we will suppose $U(x, C ; x, \bar{C})=u(x)$ (independent of $C$ ). So, on one hand,

$$
f_{0}[u(x)]=f_{0}[U(x, C ; x, \bar{C})]=f_{0}[U(x, E ; x, \emptyset)]=f[u(x)]
$$

and thus $f_{0}=f$. On the other hand,

$$
f[u(x)]=f_{0}[U(x, C ; x, \bar{C})]=f_{0}[U(x, \emptyset ; x, E)]=f_{1}\left[u_{1}(x)\right]=f_{1}(g[u(x)])
$$

and thus $f_{1}(t)=f\left[g^{-1}(t)\right]$. So, with $y=x, v=u(x), w=W(C)$, and $W_{1}(\bar{C})=$ $q[W(C)]=q(w)$, we get from (2) the functional equation

$$
f(v)=f(v w)+f\left(g^{-1}(g(v) q(w))\right) \quad(v \in[0, k[, w \in[0,1]),
$$

where $f:\left[0, k\left[\rightarrow\left[0,+\infty\left[, q:[0,1] \rightarrow[0,1]\right.\right.\right.\right.$, and $g^{-1}$ denotes the inverse of $g:[0, k[\rightarrow[0, k[$.

The solution to this equation leads to a representation that, although rank dependent in the sense that the representation depends on the preference order of the consequences, is in fact more general than the one called $R D E U$. Reference [9] explores some conditions that force the general representation to be $R D E U$.

Let $\kappa \geq-\infty, I=] \kappa,+\infty\left[, \mathbb{R}_{+}:=\right] 0,+\infty[$. We solve the following generalization of (3) (written additively):

$$
F(t)-F(t+s)=H(G(t)+Q(s)) \quad\left(t \in I, s \in \mathbb{R}_{+}\right)
$$

(cf. 10] where it is solved under different conditions) under the assumptions

(a) $F: I \rightarrow \mathbb{R}$,

(b) $G: I \rightarrow \mathbb{R}$ is strictly monotonic,

(c) $Q: \mathbb{R}_{+} \rightarrow \mathbb{R}$,

(d) $H: G(I)+Q\left(\mathbb{R}_{+}\right) \rightarrow \mathbb{R}_{+}$is strictly monotonic.

We first present the latter result and then apply it to solve the original equation (3i) . 


\section{Differentiability Properties of the solutions of Equation (4) AND A DIFFERENTIAL-FUNCTIONAL EQUATION}

Theorem 1. Suppose that equation (4) holds for all $t \in I, s \in \mathbb{R}_{+}$where the functions $F, G, Q$, and $H$ satisfy the assumptions (a)-(d). Then

(i) F is strictly decreasing,

(ii) $F$ is strictly convex or strictly concave; as such $F$ has a right derivative $F_{+}^{\prime}$ (and also a left derivative) everywhere and, except for at most countably many places, $F$ is differentiable,

(iii) $Q$ is differentiable everywhere,

(iv) $H^{-1}$ is differentiable on the open interval $J=\{F(t)-F(t+s): t \in I, s \in$ $\left.\mathbb{R}_{+}\right\}$,

(v) $G_{+}^{\prime}$ exists everywhere on $I$,

(vi) $Q^{\prime}, F_{+}^{\prime}$ and $G_{+}^{\prime}$ satisfy the differential-functional equation

$$
Q^{\prime}(s)\left[F_{+}^{\prime}(t+s)-F_{+}^{\prime}(t)\right]=G_{+}^{\prime}(t) F_{+}^{\prime}(t+s) \quad\left(t \in I, s \in \mathbb{R}_{+}\right),
$$

(vii) $G_{+}^{\prime}$ preserves sign (i.e., is everywhere positive or everywhere negative) on $I$, (viii) $Q^{\prime}$ preserves sign on $\mathbb{R}_{+}$.

Proof. By assumption (d), $H$ is a positive function; thus (i) follows from (4) immediately. For all fixed $s \in \mathbb{R}_{+}$, (4), (b), and (d) imply that the function $t \mapsto F(t)-F(t+s) \quad(t \in I)$ is strictly monotonic. Therefore $F$ is strictly Jensen convex or strictly Jensen concave. Indeed, say, in the strictly decreasing case, $F(t)-F(t+s)>F(t+s)-F((t+s)+s)$, i.e.,

$$
2 F(t+s)<F(t)+F(t+2 s) \quad\left(t \in I, s \in \mathbb{R}_{+}\right) .
$$

Furthermore, by (i), $F$ is locally bounded. Therefore, by results in 7 , Theorem 2, p. 145] or in [12, Theorem B, p. 219], $F$ is strictly convex or strictly concave, respectively. The rest of (ii) follows also from [7, Theorem 1, p. 156] and [12, Theorems B, C, pp. 4-7].

For the proof of (iii), we note that, according to (4), $F(t)-F(t+s)$ is in the codomain (in the set of function values) of $H$ for all $t \in I, s \in \mathbb{R}_{+}$. Since $H$ is strictly monotonic, we can write (4) in the form

$$
H^{-1}(F(t)-F(t+s))=G(t)+Q(s) \quad\left(t \in I, s \in \mathbb{R}_{+}\right) .
$$

Because of (i) and (ii), the set $J$ defined in (iv) is in fact an open interval of positive length in $\mathbb{R}_{+}$. On the other hand, $H^{-1}$ is strictly monotonic and so, due to Lebesgue's theorem, $H^{-1}$ is differentiable almost everywhere on $J$ (see, e.g., [5, Theorem 17.12, p. 264] or [11, pp. 5-9]). Thus, taking into consideration the properties of $F$ (obtained in (ii)), for each $s_{0} \in \mathbb{R}_{+}$, there is a point $t_{0} \in I$ such that $F$ is differentiable at $t_{0}+s_{0}$ and $H^{-1}$ is differentiable at $F\left(t_{0}\right)-F\left(t_{0}+s_{0}\right)$. Thus, while fixing $t=t_{0}$, the left-hand side of (5) is differentiable with respect to $s$ at $s_{0}$; and thus $Q$ is differentiable at $s_{0}$. This proves (iii).

Notice that we have not proved yet that $F$ or $H^{-1}$ (or $G$ ) is differentiable everywhere. We are going to prove this for $H^{-1}$ on $J$.

Let $z_{0}=F\left(t_{0}\right)-F\left(t_{0}+s_{0}\right) \in J\left(t_{0} \in I, s_{0} \in \mathbb{R}_{+}\right)$be given. Hence $s_{0}=$ $F^{-1}\left[F\left(t_{0}\right)-z_{0}\right]-t_{0}$. Since the $\operatorname{map}(t, z) \rightarrow F^{-1}[F(t)-z]-t$ is defined and jointly continuous on a neighbourhood of $\left(t_{0}, z_{0}\right)$ and $\mathbb{R}_{+}$is a neighbourhood of $s_{0}$, there exists a neighbourhood $T_{0} \times Z_{0}$ of $\left(t_{0}, z_{0}\right)$ such that $F^{-1}[F(t)-z]-t \in \mathbb{R}_{+}$for all $(t, z) \in T_{0} \times Z_{0}$. Choose in $T_{0}$ an element $t_{1}$ such that the strictly decreasing $F^{-1}$ 
is differentiable at $F\left(t_{1}\right)-z_{0}$, and let $t=t_{1}, s=F^{-1}\left[F\left(t_{1}\right)-z\right]-t_{1}$ in (55). We get

$$
H^{-1}(z)=G\left(t_{1}\right)+Q\left(F^{-1}\left(F\left(t_{1}\right)-z\right)-t_{1}\right) \quad\left(z \in Z_{0}\right) .
$$

By the differentiability of $Q$ and by the choice of $t_{1}$, the right side of (6i) is differentiable w.r.t. $z$ at $z_{0}$. This proves the differentiability of $H^{-1}$ at $z_{0}$. The point $z_{0} \in J$ being arbitrarily given, this proves (iv). Further, since $F_{+}^{\prime}$ exists everywhere, and $H^{-1}$ is differentiable on $J$, equation (5) and the chain rule yield that $G_{+}^{\prime}$ exists everywhere on $I$, as asserted in (v).

We now differentiate equation (5) with respect to $s$ and $t$ from the right to get

$$
\begin{aligned}
-\left(H^{-1}\right)^{\prime}(F(t)-F(t+s)) F_{+}^{\prime}(t+s) & =Q^{\prime}(s), \\
\left(H^{-1}\right)^{\prime}(F(t)-F(t+s))\left(F_{+}^{\prime}(t)-F_{+}^{\prime}(t+s)\right) & =G_{+}^{\prime}(t)
\end{aligned}
$$

for all $t \in I$ and $s \in \mathbb{R}_{+}$. Eliminating $\left(H^{-1}\right)^{\prime}(F(t)-F(t+s))$ from these two equations, we get the differential-functional equation (vi).

Because $F$ is strictly decreasing and strictly convex or concave, we have (see [7] Theorem 1, p. 156] and [12 Theorem B, p. 5]),

$$
F_{+}^{\prime}(t)<0 \text {, and } F_{+}^{\prime}(t+s)-F_{+}^{\prime}(t) \text { preserves its sign } \quad\left(t \in I, s \in \mathbb{R}_{+}\right) .
$$

If we had $Q^{\prime}(s) \equiv 0$, then, from (vi) and (7), $G_{+}^{\prime}(t) \equiv 0$ which is impossible since $G$ is strictly monotonic. Hence $Q^{\prime}\left(s_{1}\right) \neq 0$ for some $s_{1} \in \mathbb{R}_{+}$. Taking $s=s_{1}$ in (vi) we have (vii). Now letting $s$ vary again in (vi), we also have (viii).

\section{Solution of a general functional EQUation AND SOLUTION OF EQUATION (4)}

With the notation

(8) $\gamma(s)=Q^{\prime}(s) \quad\left(s \in \mathbb{R}_{+}\right), \quad \varphi(t)=G_{+}^{\prime}(t) \quad$ and $\quad \psi(t)=F_{+}^{\prime}(t) \quad(t \in I)$ equation (vi) is of the form

$$
\gamma(s)(\psi(t+s)-\psi(t))=\varphi(t) \psi(t+s) \quad\left(t \in I, s \in \mathbb{R}_{+}\right),
$$

where, by (7), (vii), (viii) and (8), the functions $\gamma, \psi, \varphi$ do not change signs anywhere on their domain. This general functional equation has been solved by J. Aczél, Gy. Maksa and Zs. Páles 4 in the case $I=\mathbb{R}$. Much of the argument therein cannot be applied for general $I$. Here we solve (92) on the general interval $I=] \kappa,+\infty[(\kappa \geq-\infty)$.

In order to formulate the result, we introduce the sets $P_{+}(I)$ and $P_{-}(I)$ of all pairs $(c, \mu)(c \neq 0)$ for which the function

$$
t \mapsto \mu+e^{c t} \quad(t \in I)
$$

is everywhere positive or everywhere negative, respectively. The set of pairs $(c, \mu)$ where the function (10) does not change its sign is then

$$
P(I):=P_{+}(I) \cup P_{-}(I) \text {. }
$$

Theorem 2. The functions $\varphi, \psi: I \rightarrow \mathbb{R}, \gamma: \mathbb{R}_{+} \rightarrow \mathbb{R}$ are sign preserving solutions of

$$
\gamma(s)(\psi(t+s)-\psi(t))=\varphi(t) \psi(t+s) \quad\left(t \in I, s \in \mathbb{R}_{+}\right)
$$


if, and only if, either

$$
\varphi(t)=\frac{p}{t+r}, \quad \psi(t)=\frac{q}{t+r}, \quad \gamma(s)=-\frac{p}{s} \quad\left(t \in I \neq \mathbb{R}, s \in \mathbb{R}_{+}\right)
$$

where $p, q$, and $r$ are real constants, $p q \neq 0,-r \notin I$; or

$$
\varphi(t)=\frac{a e^{c t}}{\mu+e^{c t}}, \quad \psi(t)=\frac{b}{\mu+e^{c t}}, \quad \gamma(s)=\frac{a}{1-e^{c s}} \quad\left(t \in I, s \in \mathbb{R}_{+}\right)
$$

where $a, b, c$, and $\mu$ are constants, $a b c \neq 0,(c, \mu) \in P(I)$.

Proof. Equation (9) can be rewritten as

$$
\ell(t+s)=\ell(t)+m(t) n(s) \quad\left(t \in I, s \in \mathbb{R}_{+}\right)
$$

where

$$
\ell:=1 / \psi, \quad m:=\varphi / \psi \quad \text { and } \quad n:=-1 / \gamma
$$

are again sign preserving. Since $m(t) n(s)$ is either positive for all $s$ and $t$ or negative for all $s$ and $t$, equation (14) implies that $\ell$ is strictly monotonic, and thus so is $n$. By the results of Aczél-Chung 2] (see also Járai 6]) $\ell$ has derivatives of all orders. (In [2 and 6] there are linear independence conditions which, however, are not needed for the differentiability of the function $\ell$ on the left-hand side.) This in turn implies that $m$ and $n$ in (14) have derivatives of all orders. Now we differentiate (14) with respect to $s$ and get the Pexider equation

$$
\ell^{\prime}(t+s)=m(t) n^{\prime}(s) \quad\left(t \in I, s \in \mathbb{R}_{+}\right) .
$$

Hence $\ell^{\prime}$ has the form

$$
\ell^{\prime}(t)=a_{1} e^{c t}
$$

with $a_{1} \neq 0$. Integrating we get either

$$
\ell(t)=\frac{a_{1}}{c} e^{c t}+a_{2}
$$

when $c \neq 0$; or

$$
\ell(t)=a_{1} t+a_{2}
$$

when $c=0$. Putting these back into (14) we get, for some constant $a_{3} \neq 0$,

$$
m(t)=\frac{1}{a_{3}} e^{c t}, \quad n(s)=a_{3} \frac{a_{1}}{c}\left(e^{c s}-1\right)
$$

in the case $c \neq 0$, or

$$
m(t)=\frac{1}{a_{3}}, \quad n(s)=a_{3} a_{1} s
$$

in the case $c=0$. We get (12) and (13) from (15), (20) and (21) after relabelling the constants. An easy computation shows that the functions defined in (12) and (13) indeed satisfy equation (9) and do not change sign under the restrictions given for the constants.

Theorem 3. The functions $Q, F, G$, and $H$ with the properties (a)-(d) satisfy (4) for all $t \in I$ and $s \in \mathbb{R}_{+}$if and only if either

$$
\left\{\begin{array}{l}
Q(s)=-p \ln s+C_{1}, \quad F(t)=q \ln (t+r)+B_{1}, \quad G(t)=p \ln (t+r)+A_{1}, \\
H(\xi)=-q \ln \left(1+e^{-\frac{1}{p}\left(\xi-A_{1}-C_{1}\right)}\right)
\end{array}\right.
$$


where $A_{1}, B_{1}, C_{1}$ and $p, q, r$ are constants with $p \neq 0, q<0,-r \notin I$, or

$$
F(t)=\left\{\begin{array}{ll}
\frac{\alpha}{\mu} \ln \left|\mu e^{-c t}+1\right|+B_{2} & \text { if } \mu \neq 0 \\
\alpha e^{-c t}+B_{2} & \text { if } \mu=0
\end{array} \quad(t \in I),\right.
$$

$$
H(\xi)=\left\{\begin{array}{ll}
-\frac{\alpha}{\mu} \ln \left|1-\varepsilon(c, \mu) \beta \mu e^{-\frac{1}{d}\left(\xi-A_{2}\right)}\right| & \text { if } \mu \neq 0 \\
\alpha \beta e^{-\frac{1}{d}\left(\xi-A_{2}\right)} & \text { if } \mu=0
\end{array} \quad\left(\xi \in G(I)+Q\left(\mathbb{R}_{+}\right)\right)\right.
$$

where

$$
\varepsilon(c, \mu)= \begin{cases}+1 & \text { if } \quad(c, \mu) \in P_{+}(I), \\ -1 & \text { if } \quad(c, \mu) \in P_{-}(I) .\end{cases}
$$

Here $d, \alpha, c, \beta, \mu, A_{2}, B_{2}$ are constants constrained by $d \neq 0, \beta c>0,(c, \mu) \in P(I)$, $\varepsilon(c, \mu) \alpha \beta>0$. (We have $\varepsilon(c, 0)=+1$ and so $\alpha \beta>0$ for $\mu=0$.)

Proof. Suppose that (4) holds for all $t \in I$ and $s \in \mathbb{R}_{+}$with functions $F, G, H$, and $Q$ satisfying (a)-(d). Then, by Theorem $1, Q$ is differentiable and $G_{+}^{\prime}$ and $F_{+}^{\prime}$ exist on $I$. Furthermore (9) is satisfied by the functions $\gamma, \varphi$, and $\psi$ defined in (8). Moreover, by (7), (vii), and (viii), $\gamma, \varphi$, and $\psi$ are sign preserving. Thus, by Theorem 2, we have either

$$
Q^{\prime}(s)=-\frac{p}{s}, \quad\left(s \in \mathbb{R}_{+}\right), \quad F_{+}^{\prime}(t)=\frac{q}{t+r}, \quad G_{+}^{\prime}(t)=\frac{p}{t+r} \quad(t \in I \neq \mathbb{R})
$$

with constants $p, q, r, p q \neq 0,-r \notin I$, or

$$
Q^{\prime}(s)=\frac{a}{1-e^{c s}} \quad\left(s \in \mathbb{R}_{+}\right), \quad F_{+}^{\prime}(t)=\frac{b}{\mu+e^{c t}}, \quad G_{+}^{\prime}(t)=\frac{a e^{c t}}{\mu+e^{c t}} \quad(t \in I)
$$

with constants $a, b, c, \mu, a b c \neq 0,(c, \mu) \in P(I)$. Since $F_{+}^{\prime}$ and $G_{+}^{\prime}$ are continuous, $F$ and $G$ are differentiable everywhere (see 7 , Theorem 2, p. 156]). Thus $Q, F$, and $G$ can be obtained from the equations (28) and (29) simply by integration. First we consider (28). We get by integration the asserted forms of $Q, F$ and $G$ in (22). Putting this in (4) we get

$$
q \ln \frac{t+r}{t+r+s}=q \ln \frac{(t+r) / s}{[(t+r) / s]+1}=H\left(p \ln \frac{t+r}{s}+A_{1}+C_{1}\right),
$$

and whence the form of $H$ in (22). The restriction $q<0$ follows from (17).

Next, by integrating (29) and putting the resulting forms of $Q, F, G$ into (4) to get $H$, we get the asserted forms in (23) -(27) with $d=a / c$ and $\alpha=-b / c$. (Notice that $d \ln |\beta|$ gives the constant of integration for $Q$, while $\operatorname{sign} \beta=\operatorname{sign} c$. The condition $a b c \neq 0$ is equivalent to $d \alpha \neq 0$.) The restriction $\varepsilon(c, \mu) \alpha \beta>0$ follows from the condition (d) that $H$ is positive valued. Actually, the expression within absolute value signs in (26) is positive but in this form it is easier to verify that $H$, together with (23), (24) and the first line of (25), satisfies equation (44). 
A direct computation yields that the functions thus obtained satisfy conditions (a)-(d) if , and only if, the constants satisfy the stated constraint.

\section{The solutions of equation (3)}

Let $0<k \leq+\infty, 0<k^{\prime} \leq+\infty$ be fixed. We consider (3), that is,

$$
f(v)=f(v w)+f\left(g^{-1}(g(v) q(w))\right) \quad(v \in[0, k[, w \in[0,1])
$$

under the assumptions

(A) $f:[0, k[\rightarrow[0,+\infty[$,

(B) $g:\left[0, k\left[\rightarrow\left[0, k^{\prime}[\right.\right.\right.$ is strictly monotonic and surjective,

(C) $q:[0,1] \rightarrow[0,1]$.

(Note that we allow in (B) also $k^{\prime} \neq k$, both in $\left.] 0,+\infty\right]$ ).

Theorem 4. The functions $f, g$, and $q$ with the properties $(A),(B),(C)$ satisfy (30) if and only if

- either $f \equiv 0$ on $[0, k[$ and $g, q$ are arbitrary,

- or $g$ is arbitrary and there exists a constant $c>0$ such that

$$
\begin{array}{llll}
f(0)=0, & \text { and } & f(v)=c & (v \in] 0, k[), \\
0<q(0) \leq 1, & \text { and } & q(w)=0 & (w \in] 0,1]),
\end{array}
$$

- or there exist constants $\alpha>0, c>0, d>0$ and $\mu \geq-k^{-c}$ such that

$$
q(w)=\left(1-w^{c}\right)^{d} \quad(w \in[0,1])
$$

$g(0)=f(0)=0$, and

$$
g(v)=\delta\left(\mu+v^{-c}\right)^{-d} \text { and } f(v)=\left\{\begin{array}{ll}
\frac{\alpha}{\mu} \ln \left(1+\mu v^{c}\right) & \text { if } \mu \neq 0 \\
\alpha v^{c} & \text { if } \mu=0
\end{array} \quad(v \in] 0, k[),\right.
$$

where the convention $k^{-c}=0$ if $k=+\infty$ is adapted and

$$
\begin{aligned}
& \text { if } k^{\prime}=+\infty \text {, then } \mu=-k^{-c} \text { and } \delta>0 \text { is arbitrary; } \\
& \text { if } k^{\prime}<+\infty \text {, then } \mu>-k^{-c} \text { and } \delta=k^{\prime}\left(\mu+k^{-c}\right)^{d} \text {. }
\end{aligned}
$$

Proof. If: by substitution. Only if: We distinguish the following cases.

Case 1. Suppose that $f$ is identically zero. Then (30) holds with arbitrary $g$ and $q$ satisfying (B) and (C), respectively, giving rise to the first family of solutions.

Case 2. Suppose that $f$ is not identically zero on $[0, k[$.

It will be convenient to deduce some immediate consequences of the assumptions and determine $f(0), g(0)$ beforehand and, a little later, $q(0)$ and $q(1)$. Assumption (B) implies immediately that $g(0)=g^{-1}(0)=0$. Therefore, putting $v=0$ into (30), we get $f(0)=0$. Since $f \geq 0$, (30) also implies that $f$ is increasing (since $\left.f\left(g^{-1}(g(v) q(w))\right) \geq 0\right)$-not yet strictly increasing.

We show that there exists a $\left.w_{1} \in\right] 0,1\left[\right.$ such that $q\left(w_{1}\right)<1$. Indeed, if we had $q(w)=1$ for all $w \in] 0,1[$, then (30) would give $f(v w)=0$ for all $v \in[0, k[$ and $w \in] 0,1[$, whence $f \equiv 0$ would follow. This contradicts the current case 2 assumption.

From this we shall prove that $f(v)>0$ for all $v \in] 0, k[$. Indeed, if $f$ were zero at a point $v_{0}$, then $f(v)=0$ also for all $v \in\left[0, v_{0}\left[\right.\right.$. Let $v_{1}$ be the greatest number for which $f(v)=0$ on $\left[0, v_{1}\right.$ [. Since $f$ is not identically zero, we have that $v_{1}<k$. Take a $q_{1}$ such that

$$
q\left(w_{1}\right)<q_{1}<1 \quad \text { and } \quad g\left(v_{1}\right) / q_{1}<k^{\prime}
$$


(so that $g\left(v_{1}\right) / q_{1}$ is in the domain of $g^{-1}$ ). If we had $v_{1}>0$, then (30) would yield

$$
f(v)=f\left(v w_{1}\right)+f\left(g^{-1}\left(g(v) q\left(w_{1}\right)\right)\right)=0
$$

for $v_{1}<v<\min \left(v_{1} / w_{1}, g^{-1}\left(g\left(v_{1}\right) / q_{1}\right)\right)$ because

$$
v w_{1}<v_{1} \quad \text { and } \quad g^{-1}\left(g(v) q\left(w_{1}\right)\right) \leq g^{-1}\left(g(v) q_{1}\right)<v_{1} .
$$

Thus $f$ would be identically zero on $\left[0, \min \left(v_{1} / w_{1}, g^{-1}\left(g\left(v_{1}\right) / q_{1}\right)\right)\right.$ [. Since $v_{1}<$ $v_{1} / w_{1}$ and $v_{1}<g^{-1}\left(g\left(v_{1}\right) / q_{1}\right)$, this contradicts the definition of $v_{1}$. Therefore $v_{1}=0$ and $f(v)>0$ for $\left.v \in\right] 0, k[$. (Notice, here and in the rest of case 2, the similarity to and the difference from the proof of Lemma 2 in [3].)

Putting $w=1$ in (30) and using that $f$ vanishes only at 0 , we get $q(1)=0$.

Putting $w=0$ in (30) we get

$$
f(v)=f\left(g^{-1}(g(v) q(0))\right) \quad(v \in[0, k[) .
$$

This implies that $q(0)>0$ (otherwise $f$ would be identically zero).

For $v \in] 0, k[$ and $w \in] 0,1[$ we observe the following equivalence between three statements:

$$
f \text { is constant on }[v w, v] \quad \text { iff } \quad f(v)=f(v w) \quad \text { iff } \quad q(w)=0 .
$$

The first equivalence is due to the monotonicity of $f$, and the second is due to (30) and the fact that $f$ and $g$ vanish only at 0 .

Subcase 2.1. Suppose $q\left(w_{0}\right)=0$ for some $\left.w_{0} \in\right] 0,1\left[\right.$. Let $\left.v_{0} \in\right] 0, k[$ be given. Then there exists $v$ in $] 0, k\left[\right.$ slightly greater than $v_{0}$ such that $v w_{0}<v_{0}<v$. Hence $\left[v w_{0}, v\right]$ is a neighbourhood of $v_{0}$, and by (32) $f$ is constant on this neighbourhood. This proves the local constancy of $f$ at $v_{0}$. The point $v_{0}$ in $] 0, k[$ being arbitrary, this proves the constancy of $f$ on the connected interval $] 0, k[$, say $f=c>0$ on ] $0, k[$. The constancy of $f$ on $] 0, k[$ and (32) in turn imply $q(w)=0$ for all $w \in] 0,1[$. Conversely, it is easy to check that such $f$ and $q$ indeed satisfy (30) for any function $g$ satisfying (B). This yields the second family of solutions.

Subcase 2.2. Suppose that $q$ is nowhere zero on $] 0,1[$. By (32) $f(v w) \neq f(v)$ for all $v \in] 0, k[$ and $w \in] 0,1[$. This proves that the increasing $f$ is injective on ] $0, k[$, and so is in fact strictly increasing on [0,k[. Thus, it follows from (31) that $v=g^{-1}(g(v) q(0))$, whence $q(0)=1$. Summarizing our observations thus far for the current subcase 2.2 : we have

$$
f \text { is strictly increasing on }[0, k[, \quad f(0)=g(0)=0,
$$

and

$$
q(0)=1, \quad q(w)>0 \quad \text { for } w \in] 0,1[, \quad q(1)=0 .
$$

For the time being we will restrict (30) to $v \in] 0, k[$ and $w \in] 0,1[$, and consider the boundary information in (33) and (34) only at the end.

As indicated in the Introduction, we shall solve equation (3) (which is the same as (30)) using (4).

Let $\kappa=-\ln k, I=] \kappa,+\infty[$, and define

$$
F(t)=f\left(e^{-t}\right), \quad G(t)=-\ln g\left(e^{-t}\right) \quad(t \in I),
$$

and

$$
Q(s)=-\ln q\left(e^{-s}\right), \quad H(\xi)=f\left(g^{-1}\left(e^{-\xi}\right)\right) \quad\left(s \in \mathbb{R}_{+}, \xi \in\right]-\ln k^{\prime},+\infty[) .
$$


It is straightforward to check that, as $f, g, q$ satisfy (30), (A), (B), (C), (B3), and (34), all conditions in Theorem 3 are satisfied by $F, G, H$, and $Q$; hence they must be of the forms (22)-(26). The function $Q$ in (36) cannot, however, be of the form stated in (22), because this would yield that

$$
\left.\left.q(w)=e^{-C_{1}}(-\ln w)^{p} \quad(w \in] 0,1\right]\right) .
$$

The codomain of this $q$ (both for $p>0$ and $p<0$ ) is not a subset of $[0,1]$, contrary to $(\mathrm{C})$.

Thus we only have (23), (24), (25), and (26). Taking into consideration the transformations (35)-(36) we get,

$$
g(v)=e^{-A_{2}}\left|\mu+v^{-c}\right|^{-d} \text { and } f(v)=\left\{\begin{array}{ll}
\frac{\alpha}{\mu} \ln \left|1+\mu v^{c}\right|+B_{2} & \text { if } \mu \neq 0 \\
\alpha v^{c}+B_{2} & \text { if } \mu=0
\end{array} \quad(v \in I),\right.
$$

and

$$
q(w)=\left(\frac{1-w^{c}}{\beta}\right)^{d} \quad(w \in] 0,1[),
$$

where $d, \alpha, c, \beta, \mu, A_{2}, B_{2}$, are constants with

$$
d \neq 0, \quad \varepsilon(c, \mu) \alpha \beta>0, \quad \beta c>0 \quad \text { and } \quad(c, \mu) \in P(I) .
$$

There are further restrictions to this system of constants. By comparing the functions $H$ in (26) to those obtained from (36) and (37) we get

$$
B_{2}=0, \beta=1,
$$

and thus $c>0$. Hence $\mu+e^{c t}>0$ for sufficiently large $\left.t \in I=\right] \kappa,+\infty\left[\right.$. As $\mu+e^{c t}$ preserves sign, this implies $\varepsilon(c, \mu)=1$, i.e., $\mu+e^{c t}=\mu+v^{-c}>0$ for all $\left.v \in\right] 0, k[$. By (39), $\alpha>0$ follows. The function $q$ in (38) with $\beta=1, c>0, d \neq 0$ satisfies (C) if, and only if, $d>0$. Hence the conditions in (39) are strengthened to

$$
\varepsilon(c, \mu)=1, \quad d>0, \quad \alpha>0, \quad \beta=1, \quad c>0 \quad \text { and } \mu \geq-k^{-c} .
$$

Observe that in these cases all the absolute value signs $|\cdot|$ can be replaced by parentheses $(\cdot)$ in the formulae. Taking into account the boundary information in (33)), (34), and that $g:\left[0, k\left[\rightarrow\left[0, k^{\prime}[\right.\right.\right.$ is onto, we obtain the third family of solutions.

Remark. Notice that, while we had to suppose the monotonicity and surjectivity of $g$ in order to form $g^{-1}$, no regularity (other than $f(v) \geq 0, q(w) \in[0,1]$ ) was supposed for $f$ and $q-$ similarly as in 3 . Indeed, in the case $g(v) \equiv v$, Theorem 4 is equivalent to the result in [3] as we quoted it at the beginning of this paper (the full result in [3] is somewhat more general than what we quoted).

\section{Conclusion}

With the nontrivial solutions in Theorem 4 we get from equation (2) an explicit expression for $U(x, C ; y, \bar{C})$ or, to make the expression simpler, for $\tilde{U}(x, C ; y, \bar{C}):=$ $U(x, C ; y, \bar{C})^{c}$. We write also $\tilde{u}:=u^{c}$ and $\tilde{W}:=w^{c}$, that is, $\tilde{W}(C)=W(C)^{c}$ and 
make use of $f_{0}=f, f_{1}=f \circ g^{-1}$ and $W(\bar{C})=q(W(C))$, which we obtained in the Introduction. Then we have

$$
\tilde{U}(x, C ; y, \bar{C})=\frac{\tilde{u}(x) \tilde{W}(C)+\tilde{u}(y)[1-\tilde{W}(C)]+\mu \tilde{u}(x) \tilde{u}(y) \tilde{W}(C)}{1+\mu \tilde{u}(y) \tilde{W}(C)}
$$

for all $\mu \geq-k^{-c}$. For $\mu=0$ we obtain

$$
\tilde{U}(x, C ; y, \bar{C})=\tilde{u}(x) \tilde{W}(C)+\tilde{u}(y)[1-\tilde{W}(C)],
$$

the formula for rank-dependent expected utility, $R D E U$ (with preference ranking $x \succsim y$; and a similar expression for $x \prec y)$. Notice that $\tilde{u}, \tilde{v} \in\left[0, k^{c}[, \tilde{w} \in[0,1]\right.$. It immediately follows from (41) that $\tilde{U} \in\left[0, k^{c}[\right.$ so $U \in[0, k[$. An easy calculation shows also that (40) and $c>0, \mu \geq-k^{-c}$ imply $\tilde{U} \geq 0$, thus $U \geq 0$ and, if $\tilde{u}(x) \geq \tilde{u}(y)$ for $x \succsim y$, then also $\tilde{U}<k^{c}$, thus $U<k$.

\section{REFERENCES}

[1] J. Aczél, Lectures on Functional Equations and Their Applications, Academic Press, New York/London, 1966. MR 34:8020

[2] J. Aczél and J. K. Chung, Integrable solutions of functional equations of a general type, Studia Sci. Math. Hungar. 17 (1982), 51-67. MR 85i:39008

[3] J. Aczél, R. Ger and A. Járai, Solution of a functional equation arising from utility that is both separable and additive, Proc. Amer. Math. Soc. 127 (1999), 2923-2929. CMP 99:15

[4] J. Aczél, Gy. Maksa, and Zs. Páles, Solution of a functional equation arising in an axiomatization of the utility of binary gambles, Proc. Amer. Math. Soc. (to appear). CMP 99:17

[5] E. Hewitt and K. Stromberg, Real and Abstract Analysis, Springer, New York/Heidelberg, 1975. MR 51:3363

[6] A. Járai, A remark to a paper of J. Aczél and J. K. Chung: "Integrable solutions of functional equations of a general type", Studia Sci. Math. Hungar. 19 (1984), 273-274. [MR 87m:39006

[7] M. Kuczma, An Introduction to the Theory of Functional Equations and Inequalities, Państwowe Wydawnictwo Naukowe, Warszawa/Kraków/Katowice, 1985. MR 86i:39008

[8] R. D. Luce, Coalescing, event commutativity, and theories of utility, J. Risk Uncertainty, 16 (1998), 87-114.

[9] R. D. Luce and A. A. J. Marley, Separable and additive utility of binary gambles of gains, Math. Social Sci., in press.

[10] A. Lundberg, On the functional equation $f(\lambda(x)+g(y))=\mu(x)+h(x+y)$, Aequationes Math. 16 (1977), 21-30. MR 58:29529

[11] F. Riesz and B. Szőkefalvi-Nagy, Functional Analysis, Dover, New York, 1990. MR 91g:00002

[12] A. W. Roberts and D. E. Varberg, Convex Functions, Academic Press, New York and London, 1973. MR 56:1201

Department of Pure Mathematics, University of Waterloo, Waterloo, Ontario, CANADA N2L 3G1

E-mail address: jdaczel@math.uwaterloo.ca

Current address: Institute for Mathematical Behavioral Sciences, University of California, Irvine, California 92697-5100

E-mail address: janos@aris.ss.uci.edu

Institute of Mathematics and Informatics, University of Debrecen, H-4010 Debrecen, Pf. 12, Hungary

E-mail address: maksa@math.klte.hu

Department of Pure Mathematics, University of Waterloo, Waterloo, Ontario, CANADA N2L 3G1

E-mail address: ctng@math.uwaterloo.ca

Institute of Mathematics and Informatics, University of Debrecen, H-4010 Debrecen, Pf. 12, Hungary

E-mail address: pales@math.klte.hu 